\title{
Transients of Carrier Recombination and Diffusion in Highly Excited GaN Studied by Photoluminescence and Four-Wave Mixing Techniques
}

\author{
S. JuršĖnas, S. Miasojedovas, G. KurilčIK, A. Žukauskas, \\ R. Aleksiejūnas, T. Malinauskas, M. Sūdžius \\ AND K. JARAŠIŪNAS
}

Institute of Materials Science and Applied Research, Vilnius University Saulètekio 9-III, 10222 Vilnius, Lithuania

Time-resolved photoluminescence and four-wave mixing techniques have been combined for studies of carrier relaxation dynamics in a highly photoexcited GaN epilayer. For a moderate excitation density below $1 \mathrm{~mJ} / \mathrm{cm}^{2}$, carrier recombination was due to free carrier capture by deep traps. The characteristic time of carrier capture, $\tau_{\mathrm{e}}=550 \mathrm{ps}$, was measured under deep trap saturation regime. The ambipolar diffusion coefficient for free carriers, $D=1.7 \mathrm{~cm}^{2} / \mathrm{s}$, was estimated from the analysis of the transients of the light-induced gratings of various periods. A complete saturation of the four-wave mixing efficiency was observed for the excitation energy density exceeding $1.5 \mathrm{~mJ} / \mathrm{cm}^{2}$. The latter saturation effect was shown to be related to electron-hole plasma degeneration, which results in a significant enhancement of carrier recombination rate due to onset of stimulated emission.

PACS numbers: 72.80.Ey, 72.20.Jv, 78.55.Cr

\section{Introduction}

Applications of GaN based epilayers and structures for semiconductor lasers require relevant studies of high-density carrier relaxation transients [1]. Carrier lifetime, which can be extracted from time-resolved measurements, is one of the crucial indicators of the materials quality [2,3]. However, it reflects a complex average over all the processes, which diminish the carrier density: spontaneous and 
stimulated band-to-band recombination of nonthermalized electron-hole plasma, capture of free carriers to radiative and nonradiative traps, carrier diffusion, etc. To distinguish between these processes, different time-resolved techniques must be applied.

Here we present a complementary approach based on combination of picosecond time-resolved photoluminescence (PL) and nongenerated four-wave-mixing (FWM) spectroscopy experiments, performed on a GaN epilayer at high photoexcitation conditions that were close to semiconductor laser operation regime.

\section{Experimental}

The experiments were carried out for a $2.6 \mu$ m undoped GaN sample grown at $1075^{\circ} \mathrm{C}$ by metalorganic chemical vapor deposition on a $25 \mathrm{~nm}$ thick GaN buffer layer, deposited at low temperature $\left(450^{\circ} \mathrm{C}\right)$ on sapphire (0001) substrate [4]. The samples were excited by the third or fourth harmonic (photon energy $h \nu$ was 3.55 or $4.66 \mathrm{eV}$, respectively) of the mode-locked YAG: $\mathrm{Nd}^{3+}$ (yttrium aluminum garnet) laser with pulse duration of $25 \mathrm{ps}$. The size of PL-excitation spot was $1 \mathrm{~mm}$. PL was collected in backward geometry and dispersed by a $0.4 \mathrm{~m}$ grating monochromator. A toluene optical Kerr shutter was used for temporal resolution (20 ps) of the luminescence. Carrier dynamics in GaN was investigated using the non-degenerate four-wave-mixing technique in the thin grating regime [5]. The transient free carrier gratings were recorded in the front GaN layer of the structure by two laser beams directed to the sample at a certain variable angle. This allowed us to change the grating period $\Lambda$ from 3 to $10 \mu \mathrm{m}$. The free-carrier grating decay kinetics was probed by a delayed probe beam at $1.16 \mathrm{eV}$, by measuring the intensity of its first diffraction pattern. The experiments were carried out at room temperature.

\section{Results and discussion}

Figure 1a shows some typical time-integrated PL spectra of the GaN layer for various excitation densities. The spectra are seen to contain the main emission band peaked in the vicinity of the bandgap energy $(\sim 3.4 \mathrm{eV})$, which is typical of the radiative recombination of high-density electron-hole plasma (EHP) [6, 7]. An increase in the pump energy density $\left(I_{\mathrm{p}}>1 \mathrm{~mJ} / \mathrm{cm}^{2}\right)$ leads to a carrier heating effect and an onset of stimulated transitions due to degeneration of the EHP system [7]. Since the diameter of the excited spot is much larger than the thickness of the excited region, the stimulated emission occurs mainly in the lateral direction. A part of stimulated emission is scattered due to crystal imperfections and is revealed on the low-energy edge of the emission band at the $3.3 \mathrm{eV}$ photon energy (Fig. 1). At the highest excitation levels, the stimulated emission dominates even in backward geometry. 

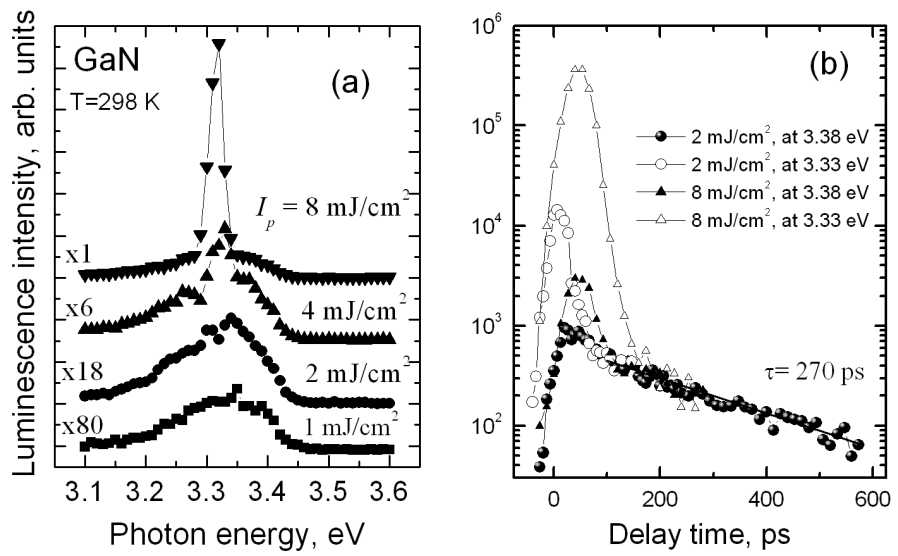

Fig. 1. (a) Time-integrated luminescence spectra of the GaN epilayer for various excitation intensities. Numbers on the right hand side, excitation energy density $\left(\mathrm{mJ} / \mathrm{cm}^{2}\right)$; those on the left hand side, sensitivity factors. The spectra are shifted vertically for clarity. (b) Luminescence transients obtained for two excitation densities $2 \mathrm{~mJ} / \mathrm{cm}^{2}$ and $8 \mathrm{~mJ} / \mathrm{cm}^{2}$, circles and triangles, respectively, and at the peak position of the spontaneous emission $(3.38 \mathrm{eV})$ and at the peak of the stimulated emission $(3.33 \mathrm{eV})$, solid and open points, respectively.

For a moderate excitation density of $I_{\mathrm{p}}=2 \mathrm{~mJ} / \mathrm{cm}^{2}$, spontaneous luminescence (solid circles in Fig. 1b) decays almost exponentially with a characteristic time constant $\tau_{\mathrm{LU}}=275 \mathrm{ps}$. The created EHP density of $10^{19} \mathrm{~cm}^{-3}$ is sufficient to maintain the deep-trap saturation regime [3]. The estimated electron capture time, $\tau_{\mathrm{e}}=2 \tau_{\mathrm{LU}}=550 \mathrm{ps}$, is typical of heteroepitaxialy grown good-quality samples [2]. Open circles in Fig. 1b show the time profile of the stimulated emission. The intensity is normalized at the 200 ps delay-time, where stimulation is expected to be exhausted. It is evident that stimulated transitions occur for a short time after the pump pulse. With an increase in the excitation density, efficient stimulated emission reduces the initial decay time to less than 10 ps (triangles in Fig. 2). The peak of intensity is reached with some delay of $50 \mathrm{ps}$. Such a delayed stimulated emission is typical of high-density nonthermalized EHP in GaN [7]. After the stimulated emission exhausted the inverse population of the carriers, luminescence is seen to exhibit a decay similar to that observed at low excitation. One can expect that at the breaking point of the luminescence decay, the carrier density is close to the EHP degeneration threshold $n_{\mathrm{th}}=5 \times 10^{18} \mathrm{~cm}^{-3}$.

Figure 2a shows the photoinduced grating transients (i.e. the dependence of diffraction efficiency $\eta$ on the probe delay time) measured at various excitation energy densities. The transients, which basically reflect changes in refraction index and, consequently, in EHP density, were found to be composed of two components. The initial component is observed at the highest pump energies used (2.8 and $\left.1.4 \mathrm{~mJ} / \mathrm{cm}^{2}\right)$. Starting at $\sim 200 \mathrm{ps}$ after the pump pulse, the initial grating 


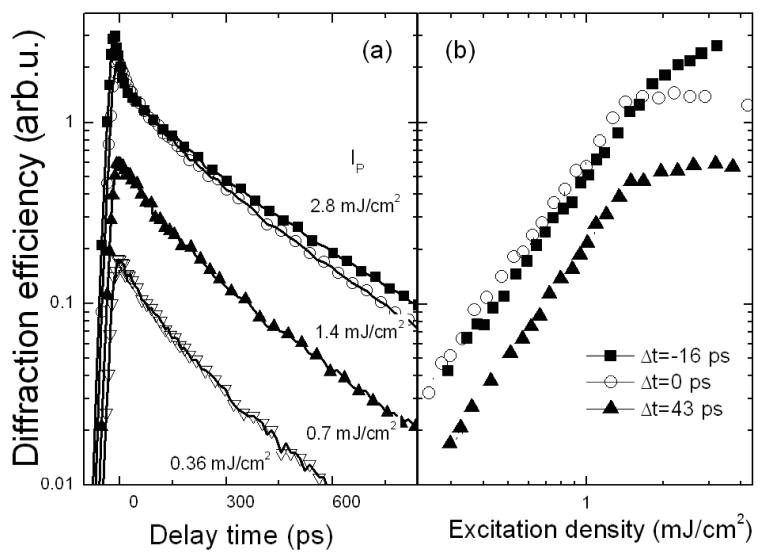

Fig. 2. (a) Photoinduced grating with a period of $\Lambda=17 \mu \mathrm{m}$ decay kinetics at various excitation energy densities, indicated on the curves. (b) Exposure characteristics measured at different probe delay times.

decay component is followed by a mono-exponential tail, i.e. $\eta(t) \sim \exp \left(-2 t / \tau_{\mathrm{G}}\right)$, where $\tau_{\mathrm{G}}$ is the grating decay time. The carrier modulation decreases due to the carrier recombination and bipolar diffusion, $\tau_{\mathrm{G}}^{-1}=\tau_{\mathrm{R}}^{-1}+4 \pi^{2} D / \Lambda^{2}$, where $\tau_{\mathrm{R}}$ is the decay time due to carrier recombination and $D$ is the coefficient of ambipolar diffusion. The measurements of $\tau_{\mathrm{G}}$ with different grating periods $\Lambda$ provided the ambipolar diffusion coefficient $D=1.7 \mathrm{~cm}^{2} / \mathrm{s}$. The obtained value of the effective recombination time, $\tau_{\mathrm{R}}=580 \mathrm{ps}$, is close to that observed in PL experiments.

The measured dependence of the diffraction efficiency as a function of excitation intensity (Fig. 2b) revealed the carrier density dependent changes of the carrier recombination rate. Almost linear carrier recombination was found at the excitation densities below $1 \mathrm{~mJ} / \mathrm{cm}^{2}$, whereas at higher excitations the exposure characteristic exhibited a tendency for saturation of the diffraction efficiency, which is in line with the PL experiments. The saturation effect was already pronounced during the action of the exciting laser pulse and increased at later times (compare the set of curves in Fig. 2b at different delay times). The onset of the saturation correlates with the manifestation of stimulated emission in the PL experiment. We attribute the saturation threshold of the FWM signal to the EHP degeneration threshold. The inverse population results in enhanced radiative emission due to stimulated recombination and, probably, enhanced diffusion of carriers due to Fermi pressure.

\section{Conclusions}

In conclusion, the performed transient PL and FWM experiments of a GaN epilayer provide important parameters of the material such as carrier lifetime and diffusion coefficient. Under conditions of strong laser excitation, a pronounced 
saturation of FWM diffraction signal has been observed and was proved to be due to reduction of carrier lifetime due to an onset of stimulated emission. The latter observation can be used for characterization of active materials for semiconductor lasers.

\section{Acknowledgments}

The research was partially sponsored by NATO's Scientific Affairs Division (Science for Peace Programme Project No. SfP-974476), European Commission (Contract No. G5MA-CT-2002-04047), and Lithuanian State Science and Studies Foundation. Prof. S. Sakai is acknowledged for providing a high quality sample. A. Ž. acknowledges the Lithuanian Ministry of Education and Science for his fellowship.

\section{References}

[1] S. Nakamura, G. Fasol, The Blue Laser Diode: GaN Based Light Emitters and Lasers, Springer, Berlin 1997.

[2] S. Juršènas, N. Kurilčik, G. Kurilčik, S. Miasojedovas, A. Žukauskas, T. Suski, P. Perlin, M. Leszczynski, P. Prystawko, I. Grzegory, Appl. Phys. Lett. 85, 952 (2004).

[3] S. Juršènas, S. Miasojedovas, G. Kurilčik, A. Žukauskas, P.R. Hageman, Appl. Phys. Lett. 83, 66 (2003).

[4] T. Wang, T. Shirahama, H.B. Sun, H.X. Wang, J. Bai, S. Sakai, H. Misawa, Appl. Phys. Lett. 76, 2220 (2000).

[5] R. Aleksiejunas, M. Sūdžius, T. Malinauskas, J. Vaitkus, K. Jarašiūnas, S. Sakai, Appl. Phys. Lett. 83, 1157 (2003).

[6] F. Binet, J.Y. Duboz, J. Off, F. Scholz, Phys. Rev. B 60, 4715 (1999).

[7] S. Juršènas, G. Kurilčik, N. Kurilčik, A. Žukauskas, P. Prystawko, M. Leszczynski, T. Suski, P. Perlin, I. Grzegory, S. Porowski, Appl. Phys. Lett. 78, 3776 (2001). 